\title{
A DIXMIER'S THEOREM FOR FINITE TYPE REPRESENTATIONS OF AMENABLE SEMIGROUPS
}

\author{
THIERRY FACK
}

\begin{abstract}
Let $S$ be an amenable locally compact semigroup. We give ergodic and spectral characterizations of the finite type representations of $S$ that are unitarizable.
\end{abstract}

\section{Introduction}

A well-known theorem of J. Dixmier (cf. [2]) asserts that any continuous and uniformly bounded Hilbert space representation of a locally compact amenable group is unitarizable. The aim of this paper is to investigate the same question for finite type representations of amenable semigroups in Hilbert spaces. More precisely, let $\pi: S \rightarrow B(H)$ be a continuous and finite type representation of an amenable semigroup $S$ in a Hilbert space $H$. We first prove, by using the so called limit isometric semigroup approach, that $\pi$ is unitarizable if and only if it is uniformly bounded and bounded away from 0 . Recall that a representation $\pi: S \rightarrow B(H)$ is bounded away from 0 if, for any non zero $x \in H$, the closure of the orbit $O(x)=\{\pi(s) x \mid s \in S\}$ of $x$ doesn't contain the zero vector. Then, by using various results on generalized $s$-numbers for von Neumann algebras (cf. [6]), we show that $\pi$ is bounded away from 0 if and only if it is unimodular, i.e. $\operatorname{Sp} \pi(s) \subset S^{1}$ for any $s \in S$. Counterexamples show that these characterizations fail if we don't assume $\pi$ of finite type. Our results give in particular structure theorems for evolution semigroups in finite von Neumann algebras, which are natural objects to study, but it is important to stress that the class of semigroups considered here also includes many interesting non commutative examples.

This paper is organized as follows: in a first section, we fix the notations. In section 2, we adapt the limit isometric approach used to discuss the unitarizability of the representations of an amenable group to the case of amenable semigroups. When the semigroup representation $\pi: S \rightarrow B(H)$ is of finite 
type, this leads to introduce two operators:

$$
R_{\pi}=\int \pi(s)^{*} \pi(s) \quad \text { and } \quad L_{\pi}=\int \pi(s) \pi(s)^{*}
$$

that we characterize geometrically. In section 3 , we first use $R_{\pi}$ and $L_{\pi}$ to get a decomposition theorem for contractive semigroup representations, which may be viewed as a generalization of the Glicksberg-DeLeeuw decomposition for finite dimensional semigroup representations (cf. [9]). Then we prove that the unitarizability of a bounded finite type representation $\pi: S \rightarrow B(H)$ is equivalent to the injectivity of $R_{\pi}$, an ergodic condition that is equivalent to the boundedness of $\pi$ away from zero. Finally, by using rearrangement inequalities in von Neuman algebras, we prove in section 4 that the boundedness of $\pi$ away from zero is equivalent to the unimodularity of $\pi$, which is a spectral condition.

We would like to thank professors Ben De Pagter and Jan van Neerven from Delft University for several valuable discussions on this paper.

\section{Notations}

\subsection{Semigroups}

A locally compact semigroup will be a locally compact Hausdorff space $S$ with an associative and separately continuous multiplication law $(s, t) \in S \times S \rightarrow$ $s t \in S$. We shall denote by $C_{b}(S)$ the $C^{*}$-algebra of all bounded continuous complex functions on $S$. For any $f \in C_{b}(S)$ and any $s \in S$, let ${ }_{s} f$ (resp. $f_{s}$ ) be the bounded continuous function defined on $S$ by ${ }_{s} f(t)=f(s t)$ (resp. $\left.f_{s}(t)=f(t s)\right)$ for any $t \in S$. A right (resp. left) invariant mean on $S$ is by definition a state $m$ on $C_{b}(S)$ such that

$$
m(f)=m\left(f_{s}\right) \quad\left(\text { resp. } m(f)=m\left({ }_{s} f\right)\right)
$$

for any $f \in C_{b}(S)$ and any $s \in S$. A locally compact semigroup $S$ will be called amenable if there exists a right invariant mean and a left invariant mean on $S$. By [11], any (discrete) abelian semigroup is amenable. For more information on amenable semigroups and interesting examples of non abelian such semigroups, we refer to [10] and [1].

\subsection{Representations}

Let $S$ be a locally compact semigroup. A continuous representation of $S$ will be a map $s \in S \rightarrow \pi(s) \in B(H)$ from $S$ to the algebra $B(H)$ of all bounded operators in a Hilbert space $H$, such that $\pi(t s)=\pi(t) \pi(s)$ for any $t, s \in S$, and that is continuous for the strong operator topology on $B(H)$. A representation $\pi$ will be called uniformly bounded if $\sup _{s \in S}\|\pi(s)\|<+\infty$. If $\pi(s)$ is unitary 
(resp. $\operatorname{Sp} \pi(s) \subset S^{1}$ ) for any $s \in S$, the representation $\pi$ will be called unitary (resp. unimodular). Finally, a representation $\pi$ will be said unitarizable if there exists a bounded invertible operator $V \in \mathrm{GL}(H)$ such that $s \rightarrow V^{-1} \pi(s) V$ is a unitary representation.

For any continuous representation $s \in S \rightarrow \pi(s) \in B(H)$ of a locally compact semigroup $S$, we shall denote by $W^{*}(\pi)$ the von Neumann subalgebra of $B(H)$ generated by the $\pi(s), s \in S$. The representation $\pi$ will be called finite type if $W^{*}(\pi)$ is a finite von Neumann algebra (see [3] for more information on finite von Neumann algebras). As in Dixmier's book [3], we shall denote by $Z(M)$ the center of the von Neumann algebra $M$ and, when $M$ is finite, by $T \in M \rightarrow T^{\#} \in Z(M)$ its canonical central trace, which is a positive ultraweakly continuous map satisfying:

$$
(T S)^{\#}=(S T)^{\#} \quad \text { for any } \quad T, S \in M .
$$

For more information on the asymptotic behavior of evolution semigroups, we refer to van Neerven's book [12], which contains a nice introduction to the limit isometric semigroup approach.

\subsection{Generalized s-numbers}

Let $M$ be a semi-finite von Neumann algebra acting on a Hilbert space $H$, and $\tau$ a semi-finite trace on $M$. For any $T \in M$, let $t \rightarrow \mu_{t}(T)$ be the non increasing rearrangement of $T$ with respect to $\tau$ (cf. [6], [7], [8]), which is defined by:

$$
\mu_{t}(T)=\inf \{\|T E\| ; E \text { is a projection in } M \text { with } \tau(1-E) \leq t\} .
$$

Recall (cf. [6], proposition 4.3, p. 323) that we have:

$$
\int_{0}^{t} \mu_{s}(T S)^{\alpha} d s \leq \int_{0}^{t} \mu_{s}(T)^{\alpha} \mu_{s}(S)^{\alpha} d s \quad \text { for any } \alpha>0 \text { and } t>0 .
$$

Note also (cf. [6], corollaire 4.2, p. 323) that the relation:

$$
\int_{0}^{t} \ln \mu_{s}(T) d s \leq \int_{0}^{t} \ln \mu_{s}(S) d s \quad \text { for any } t>0,
$$

implies $\int_{0}^{t} f\left(\mu_{s}(T)\right) d s \leq \int_{0}^{t} f\left(\mu_{s}(S)\right) d s(t>0)$ for any non decreasing continuous function $f$ on $[0,+\infty$ [ such that $t \rightarrow f(\exp (t))$ is convex. The reader will find a nice introduction to the $s$-numbers in [4]. 


\section{The limit isometric semigroup approach}

\subsection{Generalities}

Let us briefly recall the limit isometric semigroup approach used by J. Dixmier (cf. [2]) to prove that any Hilbert space representation of an amenable group is unitarizable. Let $\pi: G \rightarrow B(H)$ be a uniformly bounded continuous representation of a locally compact amenable group $G$ in a Hilbert space $H$. The existence of a right invariant mean $m_{r}$ on $G$ allows to construct a new inner product $(x, y) \rightarrow((x \mid y))$ on $H$ making all the operators $\pi(s)$ isometric. More precisely, set for any $x, y \in H$ :

$$
((x \mid y))=m_{r}(g \rightarrow\langle\pi(g) x \mid \pi(g) y\rangle),
$$

where the right hand side makes sense since $g \rightarrow\langle\pi(g) x \mid \pi(g) y\rangle$ is a bounded continuous function. We thus define a positive sesquilinear form on $H \times H$, which is continuous and satisfies:

$$
((\pi(g) x \mid \pi(g) y))=((x \mid y)) \quad \text { for any } x, y \in H \text { and any } g \in G .
$$

It is easy to check that the new inner product $(x, y) \rightarrow((x \mid y))$ defines an equivalent norm on $H$, and hence there exists a bounded invertible operator $V \in \mathrm{GL}(H)$ such that $g \rightarrow V^{-1} \pi(g) V$ is a unitary representation. From the operator theory point of view, Dixmier's approach may be summarized as follows: the inner product $(x, y) \rightarrow((x \mid y))$ constructed from an invariant mean on $G$ gives rise to a positive operator $T \in B(H)$ such that:

$$
((x \mid y))=\langle T x \mid y\rangle \quad \text { for any } x, y \in H,
$$

and the relation:

$$
((\pi(g) x \mid \pi(g) y))=((x \mid y)) \quad \text { for any } \quad x, y \in H
$$

is equivalent to:

$$
\pi(g)^{*} T \pi(g)=T \quad \text { for any } \quad g \in G .
$$

The fact that the inner product $(x, y) \rightarrow((x \mid y))$ defines an equivalent norm on $H$ implies that $T$ is invertible, a fact which allows to conjugate $\pi$ to the unitary group representation $g \rightarrow T^{1 / 2} \pi(g) T^{-1 / 2}$. We shall write symbolically $T=$ $\int \pi(g)^{*} \pi(g) d m_{r}(g)$. As we shall see now, a large part of this analysis easily extends to the case of amenable semigroups. 
2.2. The operators $R_{\pi}(X)$ and $L_{\pi}(X)$

Let $\pi: S \rightarrow B(H)$ be a uniformly bounded continuous representation of a locally compact amenable semigroup $S$ in a Hilbert space $H$. Let $m_{r}$ (resp. $m_{l}$ ) be a right (resp. left) invariant mean for $S$. For any $X \in B(H)$, define the operators:

$$
\begin{aligned}
& R_{\pi}(X)=\int \pi(s)^{*} X \pi(s) d m_{r}(s) \in B(H) \\
& L_{\pi}(X)=\int \pi(s) X \pi(s)^{*} d m_{l}(s) \in B(H)
\end{aligned}
$$

by the following formulas:

$$
\begin{aligned}
& \left\langle R_{\pi}(X) x \mid y\right\rangle=m_{r}(s \rightarrow\langle X \pi(s) x \mid \pi(s) y\rangle) \\
& \left\langle L_{\pi}(X) x \mid y\right\rangle=m_{l}\left(s \rightarrow\left\langle X \pi(s)^{*} x \mid \pi(s)^{*} y\right\rangle\right),
\end{aligned}
$$

where $x, y$ are arbitrary vectors in $H$. If $X$ is positive, it is clear that $R_{\pi}(X)$ and $L_{\pi}(X)$ are also positive. Moreover, since we have:

$$
\begin{aligned}
|\langle X \pi(s) x \mid \pi(s) y\rangle| & \leq\left(\|X\| \sup _{s \in S}\|\pi(s)\|^{2}\right)\|x\|\|y\| \\
\left|\left\langle X \pi(s)^{*} x \mid \pi(s)^{*} y\right\rangle\right| & \leq\left(\|X\| \sup _{s \in S}\|\pi(s)\|^{2}\right)\|x\|\|y\|
\end{aligned}
$$

for any $x, y \in H$ and $s \in S$, we get:

$$
\left\|R_{\pi}(X)\right\| \leq\|X\| \sup _{s \in S}\|\pi(s)\|^{2} \quad \text { and } \quad\left\|L_{\pi}(X)\right\| \leq\|X\| \sup _{s \in S}\|\pi(s)\|^{2} .
$$

Proposition 1. Let $S$ be a locally compact amenable semigroup, and $\pi: S \rightarrow B(H)$ a continuous and uniformly bounded representation of $S$ in a Hilbert space $H$. For any $X \in B(H)$, the operators:

$$
R_{\pi}(X)=\int \pi(s)^{*} X \pi(s) d m_{r}(s) \text { and } L_{\pi}(X)=\int \pi(s) X \pi(s)^{*} d m_{l}(s)
$$

satisfy:

(i) $\pi(s)^{*} R_{\pi}(X) \pi(s)=R_{\pi}(X)$ and $\pi(s) L_{\pi}(X) \pi(s)^{*}=L_{\pi}(X)$ for any $s \in S$;

(ii) $R_{\pi}(X)$ (resp. $\left.L_{\pi}(X)\right)$ belongs to the weakly closed convex hull of the $\pi(s)^{*} X \pi(s)$ (resp. of the $\left.\pi(s) X \pi(s)^{*}\right)$ where $s \in S$. In particular, if $X \in W^{*}(\pi)$, both $R_{\pi}(X)$ and $L_{\pi}(X)$ belong to $W^{*}(\pi)$;

(iii) $\left[L_{\pi}(X) R_{\pi}(X), \pi(s)\right]=0$ for any $s \in S$. 
Proof. (i) Follows immediately from the right (resp. left) invariance of $m_{r}$ (resp. of $m_{l}$ ).

(ii) Assume ad absurdio that $R_{\pi}(X)$ does not belong to the weakly closed convex hull of $\left\{\pi(s)^{*} X \pi(s) \mid s \in S\right\}$. By Hahn-Banach's theorem, there exists a weakly continuous form $\varphi$ on $B(H)$ and a real number $a$ such that $\operatorname{Re}\left[\varphi\left(\pi(s)^{*} X \pi(s)\right)\right] \leq a$ for any $s \in S$, and $\operatorname{Re}\left[\varphi\left(R_{\pi}(X)\right)\right]>a$. By [3] (théorème 1, p. 38), there exists $x_{1}, \ldots, x_{n}$ and $y_{1}, \ldots, y_{n}$ in $H$ such that:

$$
\varphi(T)=\sum_{i=1}^{n}\left\langle T x_{i} \mid y_{i}\right\rangle \quad \text { for any } \quad T \in B(H) .
$$

Since the function $s \rightarrow f(s)=\operatorname{Re} \sum_{i=1}^{n}\left\langle X \pi(s) y_{i} \mid \pi(s) y_{i}\right\rangle$ is majorized by $a$, we get:

$$
\begin{aligned}
\operatorname{Re}\left[\varphi\left(R_{\pi}(X)\right)\right] & =\operatorname{Re} \sum_{i=1}^{n}\left\langle R_{\pi}(X) x_{i} \mid y_{i}\right\rangle \\
& =\operatorname{Re} \sum_{i=1}^{n} m_{r}\left(s \rightarrow\left\langle X \pi(s) x_{i} \mid \pi(s) y_{i}\right\rangle\right) \\
& =m_{r}(f) \leq a,
\end{aligned}
$$

a contradiction. This proves that $R_{\pi}(X)$ belongs to the weakly closed convex hull of $\left\{\pi(s)^{*} X \pi(s) \mid s \in S\right\}$. In the same way, we prove that $L_{\pi}(X)$ belongs to the weakly closed convex hull of $\left\{\pi(s) X \pi(s)^{*} \mid s \in S\right\}$.

(iii) For any $s \in S$, we get from (i):

$$
\begin{aligned}
L_{\pi}(X) R_{\pi}(X) \pi(s) & =\left(\pi(s) L_{\pi}(X) \pi(s)^{*}\right) R_{\pi}(X) \pi(s) \\
& =\pi(s) L_{\pi}(X)\left(\pi(s)^{*} R_{\pi}(X) \pi(s)\right) \\
& =\pi(s) L_{\pi}(X) R_{\pi}(X) .
\end{aligned}
$$

Proposition 2. Let $S$ be a locally compact amenable semigroup and $\pi$ : $S \rightarrow B(H)$ a continuous and uniformly bounded representation of $S$ in a Hilbert space H. Set $R_{\pi}=\int \pi(s)^{*} \pi(s) d m_{r}(s)$ and denote by $u(s) \in W^{*}(\pi)$ the partial isometry in the polar decomposition of $\sqrt{R_{\pi}} \pi(s)(s \in S)$. We have:

(i) $\sqrt{R_{\pi}} \pi(s)=u(s) \sqrt{R_{\pi}}$ for any $s \in S$;

(ii) $u(s)$ is an isometry for any $s \in S \Leftrightarrow R_{\pi}$ is injective;

(iii) $\operatorname{Ker}\left(R_{\pi}\right)=\{x \in H \mid$ the norm closure of $\{\pi(s) x \mid s \in S\}$ contains zero $\}$. In particular, $R_{\pi}$ is injective if and only if $\pi$ is bounded away from zero. 
Proof. (i) For any $s \in S$, we get by proposition 1(i):

$$
\left|\sqrt{R_{\pi}} \pi(s)\right|=\sqrt{\pi(s)^{*} R_{\pi} \pi(s)}=\sqrt{R_{\pi}},
$$

and hence:

$$
\sqrt{R_{\pi}} \pi(s)=u(s)\left|\sqrt{R_{\pi}} \pi(s)\right|=u(s) \sqrt{R_{\pi}} .
$$

(ii) For any $s \in S$, we have:

$$
\operatorname{Ker}(u(s))=\operatorname{Ker}\left(\left|\sqrt{R_{\pi}} \pi(s)\right|\right)=\operatorname{Ker}\left(\sqrt{R_{\pi}}\right)=\operatorname{Ker}\left(R_{\pi}\right),
$$

and the result follows.

(iii) If the closure of $\{\pi(s) x \mid s \in S\}$ contains zero, there exists a sequence $\left(s_{n}\right)_{n \geq 1}$ in $S$ such that $\pi\left(s_{n}\right) x \rightarrow 0$ when $n \rightarrow+\infty$. Since we have:

$$
\begin{aligned}
\left\langle R_{\pi} x \mid x\right\rangle=\left|\left\langle\pi\left(s_{n}\right)^{*} R_{\pi} \pi\left(s_{n}\right) x \mid x\right\rangle\right| & =\left|\left\langle R_{\pi} \pi\left(s_{n}\right) x \mid \pi\left(s_{n}\right) x\right\rangle\right| \\
& \leq\left\|R_{\pi}\right\| \cdot\left\|\pi\left(s_{n}\right) x\right\|^{2} \rightarrow 0,
\end{aligned}
$$

we get $R_{\pi} x=0$. Conversely, if $R_{\pi} x=0$, the closure of the set $\{\pi(s) x \mid s \in S\}$ contains zero because if not there exists $a>0$ such that:

$$
\left\langle\pi(s)^{*} \pi(s) x \mid x\right\rangle=\|\pi(s) x\|^{2} \geq a \quad \text { for any } \quad s \in S,
$$

and hence $\langle X x \mid x\rangle \geq a$ for any $X$ in the convex hull of $\left\{\pi(s)^{*} \pi(s) \mid s \in\right.$ $S\}$. Then, it follows from proposition 1(ii) that $\left\langle R_{\pi} x \mid x\right\rangle \geq a$, a fact which contradicts the hypothesis $R_{\pi} x=0$. Thus, we have:

$\operatorname{Ker}\left(R_{\pi}\right)=\{x \in H \mid$ the norm closure of $\{\pi(s) x \mid s \in S\}$ contains zero $\}$, and assertion (iii) follows.

Under the assumption of theorem 2, we prove similarly that:

$\operatorname{Ker}\left(L_{\pi}\right)=\left\{x \in H \mid\right.$ the norm closure of $\left\{\pi(s)^{*} x \mid s \in S\right\}$ contains zero $\}$, where $L_{\pi}=\int \pi(s) \pi(s)^{*} d m_{l}(s)$.

We deduce also from proposition 2 that $u(s)$ is unitary for any $s \in S$ when $\pi$ is bounded away from zero and $\pi(s)$ is invertible for any $s \in S$. Indeed, since $R_{\pi}$ is injective, the operators $u(s)$ are isometries and the positive operator $\sqrt{R_{\pi}}$ has dense range. We thus have $\operatorname{Im}\left(\sqrt{R_{\pi}} \pi(s)\right)=H$, i.e. the final support of the isometry $u(s)$ is equal to one, a fact which proves that $u(s)$ is unitary.

When $\pi$ is finite type, the operators $R_{\pi}(X)$ and $L_{\pi}(X)$ share additional properties that we shall study now. 


\subsection{The finite type case}

Let $\pi: S \rightarrow B(H)$ be a uniformly bounded continuous representation of a locally compact amenable semigroup $S$ in a Hilbert space $H$. Let us show that, if $\pi$ is finite type, the maps

$$
X \in W^{*}(\pi) \rightarrow R_{\pi}(X) \in W^{*}(\pi) \quad \text { and } \quad X \in W^{*}(\pi) \rightarrow L_{\pi}(X) \in W^{*}(\pi)
$$

are projections with simple geometric characterizations. In particular, they won't depend on the choice of a right (resp. left) invariant mean $m_{r}$ (resp. $m_{l}$ ) on $S$. This result is directly inspired by [10] (theorem 3.8.4, p. 85):

THEOREM 1. Let $S$ be a locally compact amenable semigroup and $\pi: S \rightarrow$ $B(H)$ a uniformly bounded continuous representation of $S$ in the Hilbert space $H$. Assume that $\pi$ is finite type, and set:

$$
\begin{aligned}
F_{\pi}= & \left\{X \in M \mid \pi(s)^{*} X \pi(s)=X \text { for any } s \in S\right\}, \\
G_{\pi}=\left\{X \in M \mid(X Y)^{\#}=0 \text { for any } Y \in M\right. & \\
& \text { such that } \left.\pi(s) Y \pi(s)^{*}=Y \text { for any } s \in S\right\},
\end{aligned}
$$

where $M=W^{*}(\pi)$ and $X \in M \rightarrow X^{\#} \in Z(M)$ is the central trace of $M$. Then, we have:

(i) $F_{\pi}$ and $G_{\pi}$ are weakly closed linear subspaces of $M$ such that:

$$
M=F_{\pi} \oplus G_{\pi} ;
$$

(ii) The projection of $X \in M$ onto $F_{\pi}$ parallel to $G_{\pi}$ is equal to $R_{\pi}(X)$;

(iii) For any $X \in M$, the intersection of the weakly closed convex hull of the $\operatorname{set}\left\{\pi(s)^{*} X \pi(s) \mid s \in S\right\}$ with $F_{\pi}$ reduces to $\left\{R_{\pi}(X)\right\}$.

Proof. (i) and (ii). It is clear that $F_{\pi}$ and $G_{\pi}$ are weakly closed linear subspaces of $M$.Let us prove that $F_{\pi} \cap G_{\pi}=\{0\}$. For any $X \in F_{\pi} \cap G_{\pi}$, we have $\left(X X^{*}\right)^{\#}=\left(\pi(s)^{*} X \pi(s) X^{*}\right)^{\#}=\left(X \pi(s) X^{*} \pi(s)^{*}\right)^{\#}$ for any $s \in S$, and hence:

$$
\left(X X^{*}\right)^{\#}=(X Y)^{\#}
$$

for any $Y$ in the convex hull of $\left\{\pi(s) X^{*} \pi^{*}(s) \mid s \in S\right\}$.

From proposition 1(ii), we deduce that $\left(X X^{*}\right)^{\#}=\left(X L_{\pi}\left(X^{*}\right)\right)^{\#}=0$, and hence $X=0$. On the other hand, any $X \in M$ decomposes as a sum

$$
X=R_{\pi}(X)+\left(X-R_{\pi}(X)\right),
$$

where $R_{\pi}(X) \in F_{\pi}$. Let us prove that $X-R_{\pi}(X) \in G_{\pi}$. For any $Y \in M$ such that $\pi(s) Y \pi(s)^{*}=Y$ for any $s \in S$, we have:

$$
(X Y)^{\#}=\left(X \pi(s) Y \pi(s)^{*}\right)^{\#}=\left(\pi(s)^{*} X \pi(s) Y\right)^{\#} \quad \text { for any } \quad s \in S,
$$


and hence:

$$
(X Y)^{\#}=(Z Y)^{\#}
$$

for any $Z$ in the convex hull of $\left\{\pi(s)^{*} X \pi(s) \mid s \in S\right\}$.

By proposition 1(ii), we get $(X Y)^{\#}=\left(R_{\pi}(X) Y\right)^{\#}$ and hence $X-R_{\pi}(X) \in$ $G_{\pi}$. This proves (i) and (ii).

(iii) Let $X \in M$. We know by proposition 1 that the intersection of the weakly closed convex hull of $\left\{\pi(s)^{*} X \pi(s) \mid s \in S\right\}$ with $F_{\pi}$ contains $R_{\pi}(X)$. Let $Y$ be any element in this intersection. We have:

$$
X=Y+(X-Y)
$$

where $Y \in F_{\pi}$. Let us show that $X-Y \in G_{\pi}$. For any $Z \in M$ such that

$$
\pi(s) Z \pi(s)^{*}=Z \quad \text { for any } s \in S,
$$

we get as above $(X Z)^{\#}=(T Z)^{\#}$ for any $T$ in the weakly closed convex hull of $\left\{\pi(s)^{*} X \pi(s) \mid s \in S\right\}$, and hence $(X Z)^{\#}=(Y Z)^{\#}$. It follows that $X-Y \in G_{\pi}$, and $Y=R_{\pi}(X)$ by assertion (ii). The proof of (iii) is thus complete.

Under the hypothesis of theorem 1, we can prove similarly that

$$
F_{\pi}^{\prime}=\left\{X \in M \mid \pi(s) X \pi(s)^{*}=X \text { for any } s \in S\right\}
$$

and

$$
\begin{aligned}
& G_{\pi}^{\prime}=\left\{X \in M \mid(X Y)^{\#}=0 \text { for any } Y \in M\right. \text { such that } \\
& \left.\qquad \pi(s)^{*} Y \pi(s)=Y \text { for any } s \in S\right\}
\end{aligned}
$$

are supplementary weakly closed linear subspaces of $M=W^{*}(\pi)$. Moreover, the projection of $X \in M$ onto $F_{\pi}^{\prime}$ parallel to $G_{\pi}^{\prime}$ is equal to $L_{\pi}(X)$, and the intersection of the weakly closed convex hull of $\left\{\pi(s) X \pi(s)^{*} \mid s \in S\right\}$ with $F_{\pi}^{\prime}$ reduces to $\left\{L_{\pi}(X)\right\}$. It follows that, for any $X \in M$, the operators:

$$
R_{\pi}(X)=\int \pi(s)^{*} X \pi(s) d m_{r}(s) \in M
$$

and

$$
L_{\pi}(X)=\int \pi(s) X \pi(s)^{*} d m_{l}(s) \in M
$$

do not depend on the choice of the means $m_{r}$ and $m_{l}$. We shall simply write them:

$$
R_{\pi}(X)=\int \pi(s)^{*} X \pi(s) \quad \text { and } \quad L_{\pi}(X)=\int \pi(s) X \pi(s)^{*} .
$$


When $\pi$ is finite type and bounded away from zero, the partial isometries $u(s)$ in proposition 2 are in fact unitaries:

Proposition 3. Let $S$ be a locally compact amenable semigroup, and $\pi: S \rightarrow B(H)$ a continuous and uniformly bounded representation of $S$ in a Hilbert space $H$. Assume that $\pi$ is finite type and set:

$$
R_{\pi}(X)=\int \pi(s)^{*} X \pi(s) .
$$

Then, the partial isometry $u(s) \in W^{*}(\pi)$ in the polar decomposition of $\sqrt{R_{\pi}} \pi(s)$ is unitary for any $s \in S$ if and only if $\pi$ is bounded away from zero. In that case, we have:

$$
\sqrt{R_{\pi}} \pi(s)=u(s) \sqrt{R_{\pi}} \quad \text { for any } \quad s \in S,
$$

where $R_{\pi}$ is injective.

Proof. By proposition 2, we know that $\sqrt{R_{\pi}} \pi(s)=u(s) \sqrt{R_{\pi}}$ for any $s \in S$, where $R_{\pi}$ is injective if and only if $\pi$ is bounded away from zero. Since $W^{*}(\pi)$ is finite, the partial isometry $u(s)$ is unitary if and only if it is an isometry, i.e. if and only if $R_{\pi}$ is injective by proposition 2 again.

\subsection{Evolution semigroups}

For a bounded $c_{0}$ semigroup $(T(s))_{s \geq 0}$ of operators in a finite von Neumann algebra, it is possible to give a more concrete description of the operators $\int T(s)^{*} X T(s)$ and $\int T(s) X T(s)^{*}$ :

Proposition 4. Let $(T(s))_{s \geq 0}$ be a bounded $c_{0}$ semigroup of operators in a finite von Neumann algebra $M$. Then, for any $X \in M$, the averages

$$
\frac{1}{t} \int_{0}^{t} T(s)^{*} X T(s) d s \quad\left(\text { resp. } \frac{1}{t} \int_{0}^{t} T(s) X T(s)^{*} d s\right)
$$

converge in the strong operator topology to

$$
\int T(s)^{*} X T(s) \quad\left(\text { resp. } \int T(s) X T(s)^{*}\right) \quad \text { as } t \rightarrow+\infty .
$$

Proof. Let us prove that stronglim $\frac{1}{t} \int_{0}^{t} T(s)^{*} X T(s) d s=\int T(s)^{*} X T(s)$ for any $X \in M$. Since any finite von Neumann algebra is a direct sum of countably decomposable finite $W^{*}$-algebras, we may assume w.l.o.g. that $M$ has a faithful finite trace $\tau$. Let $L^{2}(M, \tau)$ be the Dixmier-Segal Hilbert space 
associated with the trace $\tau$, and consider the one parameter semigroup $(S(t))_{t \geq 0}$ of bounded linear operators in $L^{2}(M, \tau)$ defined by:

$$
S(t)(X)=T(t)^{*} X T(t) \quad\left(t \geq 0, X \in L^{2}(M, \tau)\right) .
$$

Let $E$ be the projection (in $L^{2}(M, \tau)$ ) onto the closed subspace

$$
\left\{X \in L^{2}(M, \tau) \mid T(t)^{*} X T(t)=X \text { for any } t \geq 0\right\}
$$

parallel to the closed subspace determined by the ranges of all operators $I-S(t)$ with $t \geq 0$. Since $(S(t))_{t \geq 0}$ is uniformly bounded, it follows from the ergodic theorem for continuous flows (cf. [5], corollary 3, p. 689) that we have, for any $X \in M$ :

$$
\left\|\frac{1}{t} \int_{0}^{t} T(s)^{*} X T(s) d s-E(X)\right\|_{\tau} \underset{t \rightarrow+\infty}{\longrightarrow} 0,
$$

where $\|X\|_{\tau}=\sqrt{\tau\left(X^{*} X\right)}$. Let us prove that $E(X) \in M$. Since we already know that $E(X) \in L^{2}(M, \tau)$ is a $\tau$-measurable operator affiliated with $M$, we only have to prove (cf. [8], lemma 2.5(i), p. 275) that there exists a constant $C \geq 0$ such that:

$$
\mu_{s}(E(X)) \leq C \quad \text { for almost all } s>0,
$$

where $\mu_{t}(T)$ is the $t$-th characteristic value of $T$ with respect to the trace $\tau$. For any $t>0$, set:

$$
A_{t}(X)=\frac{1}{t} \int_{0}^{t} T(s)^{*} X T(s) d s .
$$

Since $(S(t))_{t \geq 0}$ is uniformly bounded, there exists a constant $C>0$ such that $\left\|A_{t}(X)\right\| \leq C / 2$ for any $t>0$. On the other hand, we have:

$$
\left\|A_{t}(X)-E(X)\right\|_{\tau}^{2}=\int_{0}^{\infty} \mu_{s}\left(A_{t}(X)-E(X)\right)^{2} d s \underset{t \rightarrow+\infty}{\longrightarrow} 0
$$

(see [8], proposition 2.7, p. 277), and hence there exists a sequence $\left(t_{n}\right)_{n \geq 1}$ of real numbers, $t_{n} \rightarrow+\infty$, such that:

$$
\mu_{s}\left(A_{t_{n}}(X)-E(X)\right) \underset{n \rightarrow+\infty}{\longrightarrow} 0 \quad \text { for almost all } \quad s>0 .
$$

From the inequality:

$$
\begin{aligned}
\mu_{2 s}(E(X)) & \leq \mu_{s}\left(A_{t_{n}}(X)\right)+\mu_{s}\left(E(X)-A_{t_{n}}(X)\right) \\
& \leq\left\|A_{t_{n}}(X)\right\|+\mu_{s}\left(A_{t_{n}}(X)-E(X)\right),
\end{aligned}
$$


(cf. [8], lemma 2.5(v), p. 275), we immediately get $\mu_{s}(E(X)) \leq C$ for almost all $s>0$, a fact which implies that $E(X) \in M$. Then, it follows from [3] (prop. 4, p. 58) that the averages $A_{t}(X)=\frac{1}{t} \int_{0}^{t} T(s)^{*} X T(s) d s$ converge in the strong operator topology when $t \rightarrow+\infty$. Since the limit $E(X)$ obviously belongs to the weakly closed convex hull of the set $\left\{T^{*}(s) X T(s) \mid s \in S\right\}$ and satisfies $T(s)^{*} E(X) T(s)=E(X)$ for any $s \geq 0$, we finally get by theorem 1:

$$
\underset{t \rightarrow+\infty}{\text { stronglim }} \frac{1}{t} \int_{0}^{t} T(s)^{*} X T(s) d s=\int T(s)^{*} X T(s) .
$$

The relation stronglim $\lim _{t \rightarrow+\infty} \frac{1}{t} \int_{0}^{t} T(s) X T(s)^{*} d s=\int T(s) X T(s)^{*}$ is proved in a similar way.

Note that the proof of proposition 4 obviously extends to the case of discrete or continuous $k$-parameter bounded $c_{0}$-semigroups of operators in a finite von Neumann algebra.

EXAMPLES. Let us now give specific examples of bounded $c_{0}$-semigroups $(T(s))_{s \geq 0}$ of operators in the hyperfinite $I I_{1}$-factor $R$ such that the lower bound of the spectrum of $R_{T}=\int T(s)^{*} T(s)$ is arbitrary close to 0 . For any integer $N \geq 1$, set $\omega=\exp \left(\frac{2 i \pi}{N}\right)$ and, for any $t \in R$, set $\omega_{t}=\exp \left(\frac{2 i \pi t}{N}\right)$. Let $\left(e_{0}, e_{1}, \ldots, e_{N-1}\right)$ be the standard basis of $\mathrm{C}^{N}$, and fix an isomorphism $R \cong$ $R \otimes M_{N}(C)$. For any $s \geq 0$, let $T(s) \in R$ be defined by:

$$
T(s)=I \otimes Q(s) \in R \otimes M_{N}(C) \cong R,
$$

where $Q(s) \in B\left(C^{N}\right) \cong M_{N}(C)$ is given by:

$$
Q(s) e_{j}=\frac{1}{N} \sum_{k=0}^{N-1} \sum_{m=0}^{N-1} \frac{j+1}{k+1}\left(\omega^{k-j} \omega_{s}\right)^{m} e_{k} \quad(0 \leq j \leq N-1) .
$$

By straightforward calculation, we get:

$$
Q(s) Q(t)=Q(s+t) \quad \text { for } \quad s, t \geq 0,
$$

so that $(T(s))_{s \geq 0}$ is a semigroup of operators in $R$, which is obviously continuous and bounded. In fact, if $U=e^{i H}$ is the shift on $C^{N}$ given by:

$$
U\left(e_{0}\right)=e_{1}, U\left(e_{1}\right)=e_{2}, \ldots, U\left(e_{N-1}\right)=e_{0},
$$

and if $D=\operatorname{Diag}(1,1 / 2, \ldots, 1 / N)$, we have:

$$
Q(s)=D e^{i s H} D^{-1},
$$


a fact which clearly implies that $(T(s))_{s \geq 0}$ is a bounded $c_{0}$-semigroup. By straightforward calculation, we get:

$$
Q(s)^{*} e_{j}=\frac{1}{N} \sum_{k=0}^{N-1} \sum_{m=0}^{N-1} \frac{k+1}{j+1}\left(\omega^{k-j} \omega_{-s}\right)^{m} e_{k} \quad(0 \leq j \leq N-1),
$$

and hence:

$$
Q(s)^{*} Q(s) e_{j}=\frac{1}{N^{2}} \sum_{m, p, q, k=0}^{N-1} \frac{(j+1)(p+1)}{(k+1)^{2}} \omega^{m(k-j)+q(p-k)} \omega_{s}^{m} \omega_{-s}^{q} e_{p}
$$

$(0 \leq j \leq N-1)$. Since we have $\lim _{t \rightarrow+\infty} \frac{1}{t} \int_{0}^{t} \omega_{s}^{m} \omega_{-s}^{q} d s=\delta_{m}^{q}$, we get from the above formula:

$$
\lim _{t \rightarrow+\infty} \frac{1}{t} \int_{0}^{t} Q(s)^{*} Q(s) e_{j} d s=\frac{(j+1)^{2}}{N}\left(\sum_{k=0}^{N-1} \frac{1}{(k+1)^{2}}\right) e_{j},
$$

and hence:

$$
R_{T}=\int T(s)^{*} T(s)=I \otimes \operatorname{Diag}\left(\lambda_{0}, \lambda_{1}, \ldots, \lambda_{N-1}\right)
$$

by proposition 4 , where $\lambda_{j}=\frac{(j+1)^{2}}{N} \sum_{k=0}^{N-1} \frac{1}{(k+1)^{2}}(0 \leq j \leq N-1)$. It follows that:

$$
\inf \operatorname{Sp}\left(R_{T}\right)=\frac{1}{N}\left(1+\frac{1}{2^{2}}+\cdots+\frac{1}{N^{2}}\right) .
$$

Since $N$ is arbitrary, we get examples of $c_{0}$-semigroups $(T(s))_{s \geq 0}$ of operators in the hyperfinite $I I_{1}$-factor $R$ such that the lower bound of the spectrum of $R_{T}=\int T(s)^{*} T(s)$ is arbitrary close to 0 . In the same way as above, we have:

$$
S_{T}=\int T(s) T(s)^{*}=I \otimes \operatorname{Diag}\left(\mu_{0}, \mu_{1}, \ldots, \mu_{N-1}\right)
$$

where $\mu_{j}=\frac{(N+1)(2 N+1)}{6(j+1)^{2}}(0 \leq j \leq N-1)$. It follows that:

$$
S_{T}=\sqrt{R_{T}} L_{T} \sqrt{R_{T}}=\frac{(N+1)(2 N+1)}{6 N} \sum_{k=0}^{N-1} \frac{1}{(k+1)^{2}} I \otimes I,
$$

and hence:

$$
S_{T}=\sqrt{R_{T}} L_{T} \sqrt{R_{T}} \geq I .
$$

We shall see in the next section that this inequality is not an accident, but a general fact. 


\section{Ergodic results}

\subsection{A decomposition theorem for contractive semigroup representations}

Let $(T(s))_{s \geq 0}$ be a contractive $c_{0}$-semigroup on a Hilbert space $H$, and denote by $A$ its infinitesimal generator. Let $H_{0}$ be the closed subspace of all $x \in H$ such that 0 belongs to the weak closure of $\{T(s) x \mid s \geq 0\}$, and $H_{1}$ the closed linear span of all eigenvectors of $A$ with purely imaginary eigenvalues. A Glicksberg-DeLeeuw theorem asserts (see for instance [12], p. 206) that $H_{0}$ and $H_{1}$ are $T(s)$-invariant subspaces such that $H=H_{0} \oplus H_{1}$ (direct sum decomposition). Moreover, the restriction of the semigroup $(T(s))_{s \geq 0}$ to $H_{1}$ is isometric. If $H$ is finite dimensional, the Glicksberg-DeLeeuw theorem gives a decomposition of $(T(s))_{s \geq 0}$ as a sum:

$$
T(s)=T_{0}(s) \oplus T_{1}(s) \quad(s \geq 0)
$$

of a $c_{0}$-semigroup $\left(T_{0}(s)\right)_{s \geq 0}$ such that the orbit $\left\{T_{0}(s) x \mid s \geq 0\right\}$ of any $x \in H_{0}$ has 0 in its closure, and of a unitary semigroup $\left(T_{1}(s)\right)_{s \geq 0}$. The following theorem extends this result to the case of contractive finite type representations of amenable semigroups by using the limit isometric semigroup approach.

THEOREM 2. Let $S$ be a locally compact amenable semigroup, and $\pi$ : $S \rightarrow B(H)$ a continuous finite type representation of $S$ in a Hilbert space $H$. Assume that $\|\pi(s)\| \leq 1$ for any $s \in S$. Then, we have:

(i) The following closed subspaces of $H$ coincide:

$$
\{x \in H \mid \text { the norm closure of }\{\pi(s) x \mid s \in S\} \text { contains zero }\},
$$

and

$\left\{x \in H \mid\right.$ the norm closure of $\left\{\pi(s)^{*} x \mid s \in S\right\}$ contains zero $\}$.

Moreover, the orthogonal projection P on this common subspace belongs to the center of $W^{*}(\pi)$;

(ii) The representation $\pi$ splits into a direct sum of representations:

$$
\pi(s)=\left(\begin{array}{cc}
\pi_{P}(s) & 0 \\
0 & \pi_{I-P}(s)
\end{array}\right) \quad(s \in S),
$$

where: $s \rightarrow \pi_{I-P}(s)=(I-P) \pi(s)(I-P)$ is a unitary representation of $S$ in $(I-P)(H)$, and $s \rightarrow \pi_{P}(s)=P \pi(s) P$ is a representation of $S$ in $P(H)$ such that the norm closures of the orbits $O(x)=\left\{\pi_{P}(s) x \mid s \in S\right\}$ and $O^{*}(x)=\left\{\pi_{P}(s)^{*} x \mid s \in S\right\}$ of any $x \in P(H)$ both contain 0 . 
Proof. (i) For any $s \in S$, we have $\left[R_{\pi} \pi(s) \pi(s)^{*}\right]^{\#}=\left[\pi(s)^{*} R_{\pi} \pi(s)\right]^{\#}=$ $\left(R_{\pi}\right)^{\#}$, and hence $\left(R_{\pi} X\right)^{\#}=\left(R_{\pi}\right)^{\#}$ for any $X$ in the weakly closed convex hull of the set $\left\{\pi(s) \pi(s)^{*} \mid s \in S\right\}$. From proposition 1(ii), we get $\left(R_{\pi} L_{\pi}\right)^{\#}=$ $\left(R_{\pi}\right)^{\#}$. Similarly, we have $\left(R_{\pi} L_{\pi}\right)^{\#}=\left(L_{\pi}\right)^{\#}$ and hence:

$$
\left(R_{\pi}\right)^{\#}=\left(L_{\pi}\right)^{\#}=\left(R_{\pi} L_{\pi}\right)^{\#} .
$$

Since $R_{\pi}$ and $L_{\pi}$ are selfadjoint, we get from (2) and the cyclicity of the central trace:

$$
\left\{\begin{aligned}
0 & \leq\left(\left(R_{\pi}-L_{\pi}\right)^{*}\left(R_{\pi}-L_{\pi}\right)\right)^{\#}=\left(\left(R_{\pi}-L_{\pi}\right)^{2}\right)^{\#} \\
& =\left(R_{\pi}^{2}-2 R_{\pi} L_{\pi}+L_{\pi}^{2}\right)^{\#}=\left(R_{\pi}^{2}-2 R_{\pi}+L_{\pi}^{2}\right)^{\#} .
\end{aligned}\right.
$$

Since we have $\|\pi(s)\| \leq 1$ for any $s \in S$, we get from proposition 1(ii):

$$
0 \leq R_{\pi} \leq I \quad \text { and } \quad 0 \leq L_{\pi} \leq I,
$$

and hence:

$$
0 \leq R_{\pi}^{2} \leq R_{\pi} \leq I \quad \text { and } \quad 0 \leq L_{\pi}^{2} \leq L_{\pi} \leq I .
$$

From (3) and (2), we deduce that:

$$
\begin{aligned}
0 & \leq\left(\left(R_{\pi}-L_{\pi}\right)^{*}\left(R_{\pi}-L_{\pi}\right)\right)^{\#}=\left(R_{\pi}^{2}-2 R_{\pi}+L_{\pi}^{2}\right)^{\#} \\
& \leq\left(R_{\pi}-2 R_{\pi}+L_{\pi}\right)^{\#}=0,
\end{aligned}
$$

a fact which implies that $R_{\pi}=L_{\pi}$ by faithfulness of the central trace. Moreover, since we have $0 \leq\left(R_{\pi}-R_{\pi}^{2}\right)^{\#}=\left(R_{\pi}-R_{\pi} L_{\pi}\right)^{\#}=0$, we deduce that $R_{\pi}=R_{\pi}^{2}$, and hence $R_{\pi}=L_{\pi}$ is an orthogonal projection in $W^{*}(\pi)$. But we know from proposition 2(iii) that:

$\operatorname{Ker}\left(R_{\pi}\right)=\{x \in H \mid$ the norm closure of $\{\pi(s) x \mid s \in S\}$ contains zero $\}$ and

$\operatorname{Ker}\left(L_{\pi}\right)=\left\{x \in H \mid\right.$ the norm closure of $\left\{\pi(s)^{*} x \mid s \in S\right\}$ contains zero $\}$, so that these two closed subspaces of $H$ coincide and

$$
P=I-R_{\pi}=I-L_{\pi} \in W^{*}(\pi)
$$

is the orthogonal projection on this common subspace. To prove that $P$ belongs to the center of $W^{*}(\pi)$, it suffices to prove that $\operatorname{Ker}\left(R_{\pi}\right)=\operatorname{Ker}\left(L_{\pi}\right)$ is invariant 
by $\pi(s)$ and $\pi(s)^{*}$ for any $s \in S$. But if $x \in \operatorname{Ker}\left(R_{\pi}\right)$, we have:

$$
\left\|\sqrt{R_{\pi}} \pi(s) x\right\|^{2}=\left\langle\pi(s)^{*} R_{\pi} \pi(s) x \mid x\right\rangle=\left\langle R_{\pi} x \mid x\right\rangle=0,
$$

and hence $\pi(s) x \in \operatorname{Ker}\left(R_{\pi}\right)$. On the other hand, we have:

$$
\left\|\sqrt{R_{\pi}} \pi(s)^{*} x\right\|^{2}=\left\langle\pi(s) L_{\pi} \pi(s)^{*} x \mid x\right\rangle=\left\langle L_{\pi} x \mid x\right\rangle=\left\langle R_{\pi} x \mid x\right\rangle=0,
$$

and hence $\pi(s)^{*} x \in \operatorname{Ker}\left(R_{\pi}\right)$. This shows that $\operatorname{Ker}\left(R_{\pi}\right)$ is invariant by $\pi(s)$ and $\pi(s)^{*}$ for any $s \in S$, and (i) is proved.

(ii) Since $P$ belongs to the center of $W^{*}(\pi)$, we only have to prove that $s \rightarrow \pi_{I-P}(s)=(I-P) \pi(s)(I-P)$ is a unitary representation of $S$ in $(I-P)(H)$. For any $s \in S$, let $u(s) \in W^{*}(\pi)$ be the partial isometry in the polar decomposition of $\sqrt{R_{\pi}} \pi(s)$. We know from proposition 2(i) that:

$$
\sqrt{R_{\pi}} \pi(s)=u(s) \sqrt{R_{\pi}},
$$

and hence:

$$
\begin{aligned}
\pi_{I-P}(s) & =(I-P) \pi(s)(I-P)=\sqrt{R_{\pi}} \pi(s)(I-P) \\
& =u(s) \sqrt{R_{\pi}}(I-P)=(I-P) u(s)(I-P) .
\end{aligned}
$$

It follows that, for any $s \in S, \pi_{I-P}(s)$ is a partial isometry in the reduced von Neumann algebra $W^{*}(\pi)_{I-P}$ with initial support $\operatorname{supp}\left(\sqrt{R_{\pi}}\right)(I-P)=$ $(I-P)$. Since $W^{*}(\pi)_{I-P}$ is a finite von Neumann algebra, this implies that $\pi_{I-P}(s)$ is a unitary for any $s \in S$, and the proof of (ii) is complete.

EXAMPLE 1. For contractive $c_{0}$-semigroups $(T(s))_{s \geq 0}$ of operators in a finite von Neumann algebra, we get from theorem 2 a direct sum decomposition that goes beyond the Glicksberg-DeLeeuw theorem. Indeed, let $A$ be the infinitesimal generator of $(T(s))_{s \geq 0}$, and denote by $H_{0}$ the closed subspace of all $x \in H$ such that 0 belongs to the norm closure of $\{T(s) x \mid s \geq 0\}$. Let $H_{1}$ be the orthogonal complement of $H_{0}$ in $H$. By theorem 2, the restriction of $-i A$ to $H_{1}$ is a selfadjoint operator on $H_{1}$ and, by splitting its spectrum into a continuous part and a pure point part, we get an orthogonal decomposition $H_{1}=H_{1, \text { cont }} \oplus H_{1, \text { pp }}$. We deduce a splitting:

$$
T(s)=T_{0}(s) \oplus T_{1, \text { cont }}(s) \oplus T_{1, \mathrm{pp}}(s) \quad(s \geq 0),
$$

where the $c_{0}$-semigroup $T_{0}$ has the property that 0 is in the norm closure of the orbit $\left\{T_{0}(s) x \mid s \geq 0\right\}$ of any $x \in H_{0}$, and where $T_{1}=T_{1, \text { cont }} \oplus T_{1 \text {,pp }}$ is a unitary $c_{0}$-semigroup. When $H_{1, \mathrm{pp}}=0$, the $c_{0}$-semigroup $T_{1, \mathrm{pp}}$ in the 
Glicksberg-DeLeeuw decomposition is trivial. However, it is still possible, thanks to theorem 2 , to extract from $T$ a unitary part $T_{1}$ which corresponds in this case to the continuous spectrum of $-i A$.

As a corollary, we get from theorem 2:

Corollary 1. Let $S$ be a locally compact amenable semigroup, and $\pi$ : $S \rightarrow B(H)$ be a continuous finite type representation of $S$ in a Hilbert space $H$. The following conditions are equivalent:

(i) $\pi$ is a unitary representation;

(ii) There exists $a>0$ such that $\alpha I \leq \pi(s)^{*} \pi(s) \leq I$ for any $s \in S$;

(iii) $\|\pi(s)\| \leq 1$ for any $s \in S$, and $\pi$ is bounded away from 0 .

Proof. (i) $\Rightarrow$ (ii) $\Rightarrow$ (iii) is obvious. Let us prove (iii) $\Rightarrow$ (i). Since $\pi$ is bounded away from 0 , we have $P=0$ in theorem 2 , and hence $\pi$ is a unitary representation.

So, for any finite type continuous semigroup representation $\pi: S \rightarrow B(H)$ such that $\|\pi(s)\| \leq 1$, the operator $\sqrt{R_{\pi}}$ is a projection whose kernel is trivial if and only if $\pi$ is bounded away from 0 . The condition $\sqrt{R_{\pi}}=I$, which means that $\pi$ is unitary, is thus equivalent to the boundedness of $\pi$ away from 0 . Let us now extend this observation to uniformly bounded finite type representations.

\subsection{An ergodic characterization}

The following theorem characterizes the finite type representations of a locally compact amenable semigroup in a Hilbert space that are unitarizable:

THeOREM 3. Let $S$ be a locally compact amenable semigroup, and $\pi$ : $S \rightarrow B(H)$ be a continuous finite type representation of $S$ in a Hilbert space $H$. The following conditions are equivalent:

(i) $\pi$ is unitarizable;

(ii) There exist a, $\beta$ with $0<a \leq \beta$ such that $\alpha I \leq \pi(s)^{*} \pi(s) \leq \beta I$ for any $s \in S$;

(iii) $\pi$ is uniformly bounded and bounded away from 0 .

Moreover, if one of these conditions is fulfilled, there exists a positive invertible element $V \in W^{*}(\pi)$ with $\|V\| \leq \sup _{s \in S}\|\pi(s)\|$ such that the representation $s \rightarrow V^{-1} \pi(s) V$ is unitary.

The proof of this theorem is essentially a refinement, based on the following proposition, of the argument used in the proof of theorem 2 .

Proposition 5. Let $S$ be a locally compact amenable semigroup and $\pi$ : $S \rightarrow B(H)$ be a continuous, uniformly bounded and finite type representation 
of $S$ in a Hilbert space $H$. Set $S_{\pi}=\sqrt{R_{\pi}} L_{\pi} \sqrt{R_{\pi}}$, where:

$$
R_{\pi}=\int \pi(s)^{*} \pi(s) \quad \text { and } \quad L_{\pi}=\int \pi(s) \pi(s)^{*} .
$$

Then the spectral projection of $S_{\pi}$ corresponding to the interval ]0, 1[ is zero.

In other words, the operator $S_{\pi}=\sqrt{R_{\pi}} L_{\pi} \sqrt{R_{\pi}}$, which is a projection when $\|\pi(s)\| \leq 1$ for any $s \in S$, has no spectrum between 0 and 1 in the general case. The proof of this assertion is based on the following lemma, which has its own interest:

Lemma 1. Let $S$ be a locally compact amenable semigroup and $\pi: S \rightarrow$ $B(H)$ be a continuous, uniformly bounded and finite type representation of $S$ in a Hilbert space $H$. Then, for any $X$ in the commutative von Neumann subalgebra of $W^{*}(\pi)$ generated by the operator $S_{\pi}=\sqrt{R_{\pi}} L_{\pi} \sqrt{R_{\pi}}$, we have:

$$
\left(R_{\pi} X\right)^{\#}=\left(S_{\pi} X\right)^{\#} \quad \text { and } \quad\left(L_{\pi} \sqrt{R_{\pi}} X \sqrt{R_{\pi}} L_{\pi}\right)^{\#}=\left(S_{\pi} X S_{\pi}\right)^{\#} .
$$

Proof. Note first that we have, for any integer $n \geq 0$ :

$$
\left[R_{\pi}\left(L_{\pi} R_{\pi}\right)^{n}\right]^{\#}=\left[L_{\pi}\left(L_{\pi} R_{\pi}\right)^{n}\right]^{\#}=\left[\left(L_{\pi} R_{\pi}\right)^{n+1}\right]^{\#} .
$$

Indeed, we have $\pi(s) L_{\pi} R_{\pi}=L_{\pi} R_{\pi} \pi(s)$ for any $s \in S$ by proposition 1(iii), and it follows from the cyclicity of the central trace that:

$$
\begin{aligned}
{\left[R_{\pi}\left(L_{\pi} R_{\pi}\right)^{n}\right]^{\#}=\left[\pi(s)^{*} R_{\pi} \pi(s)\left(L_{\pi} R_{\pi}\right)^{n}\right]^{\#} } & =\left[\pi(s)^{*} R_{\pi}\left(L_{\pi} R_{\pi}\right)^{n} \pi(s)\right]^{\#} \\
& =\left[R_{\pi}\left(L_{\pi} R_{\pi}\right)^{n} \pi(s) \pi(s)^{*}\right]^{\#} .
\end{aligned}
$$

By normality of the central trace, we get $\left[R_{\pi}\left(L_{\pi} R_{\pi}\right)^{n}\right]^{\#}=\left[R_{\pi}\left(L_{\pi} R_{\pi}\right)^{n} Y\right]^{\#}$ for any $Y$ in the closed convex hull of $\left\{\pi(s) \pi(s)^{*} \mid s \in S\right\}$, and hence:

$$
\left[R_{\pi}\left(L_{\pi} R_{\pi}\right)^{n}\right]^{\#}=\left[R_{\pi}\left(L_{\pi} R_{\pi}\right)^{n} L_{\pi}\right]^{\#}=\left[\left(L_{\pi} R_{\pi}\right)^{n+1}\right]^{\#} .
$$

In the same way, we get $\left[L_{\pi}\left(L_{\pi} R_{\pi}\right)^{n}\right]^{\#}=\left[\left(L_{\pi} R_{\pi}\right)^{n+1}\right]^{\#}$, and (4) follows.

To prove the lemma, we may and do assume by elementary spectral theory that $X=S_{\pi}^{n}$ with $n \geq 0$. We then have:

$$
\begin{aligned}
\left(R_{\pi} X\right)^{\#}=\left(\sqrt{R_{\pi}} S_{\pi}^{n} \sqrt{R_{\pi}}\right)^{\#} & =\left[R_{\pi}\left(L_{\pi} R_{\pi}\right)^{n}\right]^{\#}=\left[\left(L_{\pi} R_{\pi}\right)^{n+1}\right]^{\#} \\
& =\left(S_{\pi}^{n+1}\right)^{\#}=\left(S_{\pi} X\right)^{\#},
\end{aligned}
$$

and:

$$
\begin{aligned}
\left(L_{\pi} \sqrt{R_{\pi}} X \sqrt{R_{\pi}} L_{\pi}\right)^{\#} & =\left[\left(L_{\pi} R_{\pi}\right)^{n+1} L_{\pi}\right]^{\#}=\left[L_{\pi}\left(L_{\pi} R_{\pi}\right)^{n+1}\right]^{\#} \\
& =\left[\left(L_{\pi} R_{\pi}\right)^{n+2}\right]^{\#}=\left(S_{\pi}^{n+2}\right)^{\#}=\left(S_{\pi} X S_{\pi}\right)^{\#},
\end{aligned}
$$


so that the lemma is proved.

ProOf of Proposition 5. Let us denote by $E$ the spectral projection of $S_{\pi}$ corresponding to the interval $] 0,1[$. To prove that $E=0$, it suffices to show that we have:

$$
S_{\pi} E=S_{\pi}^{2} E .
$$

Indeed, since we have $S_{\pi}^{2} E \leq S_{\pi} E$, the relation (5) is equivalent to $E=0$ by elementary spectral theory. To prove (5), consider the vector state $\omega_{x}$ on the center of $W^{*}(\pi)$ associated with a norm one vector $x \in H$, and denote by $\tau_{x}$ the state on $W^{*}(\pi)$ defined by:

$$
\tau_{x}(T)=\omega_{x}\left(T^{\#}\right) \quad\left(T \in W^{*}(\pi)\right) .
$$

We have:

$$
\begin{aligned}
\omega_{x}\left(\left(S_{\pi} E\right)^{\#}\right)^{2} & =\omega_{x}\left(\left(E S_{\pi} E\right)^{\#}\right)^{2}=\tau_{x}\left(\left(E \sqrt{R_{\pi}} L_{\pi}\right)\left(\sqrt{R_{\pi}} E\right)\right)^{2} \\
& \leq \tau_{x}\left(E \sqrt{R_{\pi}} L_{\pi}^{2} \sqrt{R_{\pi}} E\right) \tau_{x}\left(E R_{\pi} E\right) \quad(\text { Cauchy-Schwarz }) \\
& =\omega_{x}\left(\left(E \sqrt{R_{\pi}} L_{\pi}^{2} \sqrt{R_{\pi}} E\right)^{\#}\right) \omega_{x}\left(\left(E R_{\pi} E\right)^{\#}\right) \\
& =\omega_{x}\left(\left(L_{\pi} \sqrt{R_{\pi}} E \sqrt{R_{\pi}} L_{\pi}\right)^{\#}\right) \omega_{x}\left(\left(R_{\pi} E\right)^{\#}\right) \\
& =\omega_{x}\left(\left(S_{\pi}^{2} E\right)^{\#}\right) \omega_{x}\left(\left(S_{\pi} E\right)^{\#}\right) \quad(\text { Lemma } 1),
\end{aligned}
$$

and hence:

$$
\omega_{x}\left(\left(S_{\pi} E\right)^{\#}\right) \leq \omega_{x}\left(\left(S_{\pi}^{2} E\right)^{\#}\right)
$$

for any norm one vector $x \in H$. It follows that $\left(S_{\pi} E\right)^{\#} \leq\left(S_{\pi}^{2} E\right)^{\#}$ and, since we have $S_{\pi}^{2} E \leq S_{\pi} E$, we get $\left(S_{\pi} E\right)^{\#}=\left(S_{\pi}^{2} E\right)^{\#}$ and $\left(S_{\pi} E-S_{\pi}^{2} E\right)^{\#}=0$. By faithfulness of the central trace, we finally get $S_{\pi} E=S_{\pi}^{2} E$, and (5) is proved.

We are now in position to prove theorem 3.

Proof of THEOREM 3. (i) $\Rightarrow$ (ii) $\Rightarrow$ (iii) is obvious.

(iii) $\Rightarrow$ (i) For any $s \in S$, let $u(s) \in W^{*}(\pi)$ be the partial isometry in the polar decomposition of $\sqrt{R_{\pi}} \pi(s)$. Since $\pi$ is bounded away from 0 , we know by proposition 3 that $u(s)$ is unitary and $\operatorname{Ker}\left(R_{\pi}\right)=0$. Let us prove that $V=\sqrt{R_{\pi}} \in W^{*}(\pi)$ is invertible. Since we have $V \pi(s)=u(s) V$ for any $s \in S$, this will imply that $\pi(s)=V^{-1} u(s) V$ and $\pi$ will be unitarizable. Since we obviously have $\|V\| \leq \sup _{s \in S}\|\pi(s)\|$, the proof of the theorem will be complete. To prove that $V=\sqrt{R_{\pi}} \in W^{*}(\pi)$ is invertible, it suffices to prove the stronger condition:

$$
S_{\pi}=\sqrt{R_{\pi}} L_{\pi} \sqrt{R_{\pi}} \geq 1,
$$


i.e. the spectral projection of $S_{\pi}$ corresponding to the interval [0,1[ is zero. By proposition 5, we only have to prove that the orthogonal projection $E_{0}$ on $\operatorname{Ker}\left(S_{\pi}\right)$ is zero. But we have from lemma 1:

$$
0=\left(S_{\pi} E_{0}\right)^{\#}=\left(R_{\pi} E_{0}\right)^{\#}=\left(\left|\sqrt{R_{\pi}} E_{0}\right|^{2}\right)^{\#},
$$

and hence $\sqrt{R_{\pi}} E_{0}=0$ by faithfulness of the central trace. Since $\operatorname{Ker}\left(R_{\pi}\right)=$ 0 , we get $E_{0}=0$.

REMark 1. When $\|\pi(s)\| \leq 1$, the proof of theorem 2 shows that $R_{\pi}$ is an invertible projection, and hence $R_{\pi}=S_{\pi}=I$. For a general uniformly bounded finite type representation $\pi: S \rightarrow B(H)$ which is bounded away from 0 , we know that $R_{\pi}$ is invertible and $S_{\pi}=\sqrt{R_{\pi}} L_{\pi} \sqrt{R_{\pi}} \geq I$, but it is no longer true that $R_{\pi}=S_{\pi}=I$. The examples of $c_{0}$-semigroups of operators of the hyperfinite $I I_{1}$-factor given in section 2.4 show that the spectrum of $R_{\pi}$ could be arbitrary close to 0 , while $S_{\pi} \in W^{*}(\pi)$ is strictly greater than $I$.

Remark 2. Theorem 3 is false if the representation $\pi$ is not of finite type. Indeed, let $\pi$ be the representation of $R_{+}$given by the bounded $c_{0}$-semigroup $(T(t))_{t \geq 0}$ of operators in $H=L^{2}\left(R_{+}\right)$defined by:

$$
\left(T_{t} f\right)(x)=1_{[t,+\infty[}(x) f(x-t) \quad\left(x \in R_{+}, \quad f \in L^{2}\left(R_{+}\right)\right) .
$$

Since $T_{t}$ is an isometry, condition (ii) in theorem 3 is satisfied. However, since we have $T_{t}^{*}(f)=e^{-t} f$ for $f(x)=e^{-x}$, the spectrum of $T_{t}$ is not always contained in $S^{1}$, and the representation $\pi$ is not unitarizable. But here, $\pi$ is not of finite type, because an isometry in a finite von Neumann algebra is automatically unitary.

Corollary 2. Let $(T(t))_{t \geq 0}$ be a bounded $c_{0}$-semigroup of operators in a finite von Neumann algebra $M$, and denote by $A$ its infinitesimal generator. The following conditions are equivalent:

(i) $(T(t))_{t \geq 0}$ is bounded away from 0 ;

(ii) There exists a positive invertible element $V \in W^{*}(\pi)$ such that $-i V^{-1} A V$ is a selfadjoint operator.

Proof. (i) $\Rightarrow$ (ii) By theorem 3, there exists a positive invertible element $V \in W^{*}(\pi)$ such that $V^{-1} T(t) V$ is unitary for any $t \geq 0$. Since $-i$ times the generator of a $c_{0}$-semigroup of unitaries is selfadjoint, the result follows.

(ii) $\Rightarrow$ (i) If there exists a positive invertible element $V \in W^{*}(\pi)$ such that the operator $P=-i V^{-1} A V$ is a selfadjoint operator, we get:

$$
\left.\frac{d}{d t}\left(V e^{i t P} V^{-1}\right)\right|_{t=0}=i V P V^{-1}=A,
$$


and hence $T(t)=V e^{i t P} V^{-1}$ for any $t \geq 0$ by uniqueness of the Cauchy problem associated with $A$. The result immediately follows.

\section{Spectral results}

\subsection{A spectral inequality}

In this section, we give a spectral characterization of the finite type representations of a locally compact amenable semigroup that are unitarizable. This characterization is based on a spectral inequality for unimodular elements in a semi-finite von Neumann algebra that has its own interest. Recall that an element $X$ in a von Neumann algebra $M$ is called unimodular if $\operatorname{Sp}(X) \subset \mathrm{S}^{1}$. It was proved by B. Russo (cf. [13], lemma 2.2, p. 165) that any unimodular contraction in a finite von Neumann algebra is unitary. The following inequality, which is directly inspired by Russo's result, shows that we have in particular $\tau(E) \leq \tau\left(E|X|^{2} E\right)$ for any unimodular element $X$ in a von Neumann algebra $M$ equipped with a normal semi-finite trace $\tau$, and any finite projection $E \in M$ whose image is stable by $X$ :

THeOREM 4. Let $M$ be a von Neumann algebra equipped with a normal semifinite trace $\tau$. Let $X$ be a unimodular element in $M$. For any continuous function $f$ on $[0,+\infty[$ such that $t \rightarrow f(\exp (t))$ is convex and any projection $E \in M$ such that $X E=E X E$ and $\tau(E)<+\infty$, we have:

$$
t f(1) \leq \int_{0}^{t} f\left(\mu_{s}(E X E)\right) d s \quad \text { for any } t \in[0, \tau(E)] .
$$

In particular, we have $\tau(E) \leq \tau\left(E|X|^{2} E\right)$.

Proof. Let us denote by $H$ the Hilbert space upon which $M$ acts. To prove theorem 4, we may assume w.l.o.g. that $E \neq 0$. Moreover, by using [3] (corollaire 2, p. 83), we may assume in addition that $\tau$ is faithful.

First step. Let us first show that $E X E$ is a unimodular element in the reduced von Neumann algebra $M_{E}$. Since $X$ is unimodular, there exists for any complex number $\lambda$ with $|\lambda| \neq I$ an element $Y \in M$ such that:

$$
(X-\lambda I) Y=Y(X-\lambda I)=I .
$$

From the relation $E Y E(E X E-\lambda E)=E Y(X E-\lambda E)=E Y(X-\lambda I) E=E$, we deduce that the operator $E X E-\lambda E: E(H) \rightarrow E(H)$ is injective with closed range. Let $P$ be the orthogonal projection on $\operatorname{Im}(E X E-\lambda E)$. Since $P$ is equivalent to $\operatorname{supp}(E X E-\lambda E)=E$ in the von Neumann algebra $M_{E}$ which is finite, we get $P=E$, and hence $E X E-\lambda E$ is invertible in $M_{E}$. This proves that $E X E$ is a unimodular element in $M_{E}$. 
Second step. Let us now prove that we have, for any unimodular element $X$ in $M_{E}$ :

$$
0 \leq \int_{0}^{t} \ln \mu_{s}(X) d s \quad \text { for any } \quad t \in[0, \tau(E)],
$$

with equality for $t=\tau(E)$. Here, the generalized $s$-number $\mu_{s}(X)$ is associated with the reduced trace $\tau_{E}$ on $M_{E}$. Since $E \neq 0$, we have $\tau(E)>0$ and, by replacing $\tau$ by $\frac{\tau}{\tau(E)}$ if necessary, we may and do assume that $\tau(E)=1$. Since $X$ is unimodular, we have $\operatorname{Sp}\left(X^{-1}\right) \subset \mathrm{S}^{1}$ and the spectral radius of $X^{-1}$ is one. For any $\varepsilon>0$, we thus may choose an integer $n \geq 1$ such that $\left\|X^{-n}\right\| \leq(1+\varepsilon)^{n}$. For any $\alpha>0$ and $0<t<1$, we have:

$$
\begin{aligned}
1=\int_{0}^{t} \mu_{s}\left(X^{n} X^{-n}\right)^{\alpha} \frac{d s}{t} & \leq \int_{0}^{t} \mu_{s}\left(X^{n}\right)^{\alpha}(1+\varepsilon)^{n \alpha} \frac{d s}{t} \\
& =(1+\varepsilon)^{n \alpha} \int_{0}^{t} \mu_{s}\left(X^{n}\right)^{\alpha} \frac{d s}{t}
\end{aligned}
$$

and hence, by using [6] (corollaire 4.4, p. 324):

$$
1 \leq(1+\varepsilon)^{n \alpha} \int_{0}^{t} \mu_{s}(X)^{n \alpha} \frac{d s}{t} .
$$

Taking the power $1 / \alpha$ and letting $\alpha \rightarrow 0$, we get:

$$
1 \leq(1+\varepsilon)^{n} \exp \int_{0}^{t} \ln \left(\mu_{s}(X)^{n}\right) \frac{d s}{t}=\left((1+\varepsilon) \exp \left(\int_{0}^{t} \ln \mu_{s}(X) \frac{d s}{t}\right)\right)^{n} .
$$

Letting $\varepsilon \rightarrow 0$, we finally get $1 \leq \exp \left(\int_{0}^{t} \ln \mu_{s}(X) \frac{d s}{t}\right)$, and hence:

$$
0 \leq \int_{0}^{t} \ln \mu_{s}(X) d s \quad \text { for } \quad 0<t<1 .
$$

This relation is still true for $t=0$ and, since the Fuglede-Kadison determinant $\Delta(X)$ of $X$ is less than the spectral radius of $X$ which is one by [3] (théorème 10, (v), p. 109), we get:

$$
0 \leq \int_{0}^{1} \ln \mu_{s}(X) d s \leq 0,
$$

so that (7) is proved.

Third step. We are now in position to prove theorem 4 . Let $X$ be a unimodular element in $M$ and consider a finite projection $E \in M$ such that $X E=E X E$. Since $E X E \in M_{E}$ is unimodular, we get from relation (7):

$$
0 \leq \int_{0}^{t} \ln \mu_{s}(E X E) d s \quad \text { for } \quad 0 \leq t \leq \tau(E),
$$


with equality for $t=\tau(E)$ and where, thanks to [6] (proposition 1.5, p. 312), $\mu_{s}(E X E)$ is also the generalized $s$-number associated with the trace $\tau$. By [6] (corollaire 4.2, p. 323), we get for any continuous function $f$ on $[0,+\infty$ [ such that $t \rightarrow f(\exp (t))$ is convex:

$$
t f(1)=\int_{0}^{t} f\left(\mu_{s}(I)\right) d s \leq \int_{0}^{t} f\left(\mu_{s}(E X E)\right) d s \quad \text { for } \quad 0 \leq t \leq \tau(E) .
$$

Taking $f(s)=s^{2}$ and $t=\tau(E)$, we get:

$$
\tau(E) \leq \int_{0}^{\tau(E)} \mu_{s}(E X E)^{2} d s=\tau\left(|E X E|^{2}\right) \leq \tau\left(E X^{*} X E\right),
$$

and the proof of the theorem is complete.

From theorem 4, we deduce the following result of [13]:

COROLlaRY 3. Let $M$ be a finite von Neumann algebra acting on a separable Hilbert space. Any contraction in $M$ with spectrum contained in the unit circle is unitary.

Proof. Let $\tau$ be a faithful finite trace on $M$. For any contraction $T$ in $M$, we have $\mu_{t}(T) \leq 1$, and hence $\ln \mu_{t}(T)=0$ for $0 \leq t<\tau(I)$ by theorem 4 . This implies that $|T|=I$, and $T$ is unitary.

4.2. Spectral characterization of unitarizable finite type representations of amenable semigroups

We are now in position to prove the following result:

THeORem 5. Let $S$ be a locally compact amenable semigroup, and $\pi$ : $S \rightarrow B(H)$ be a continuous finite type representation of $S$ in a Hilbert space $H$. The following conditions are equivalent:

(i) $\pi$ is unitarizable;

(ii) There exist a, $\beta$ with $0<a \leq \beta$ such that $\alpha I \leq \pi(s)^{*} \pi(s) \leq \beta I$ for any $s \in S$;

(iii) $\pi$ is uniformly bounded and unimodular.

Proof. We already know the equivalence of (i) and (ii) from theorem 3. Since (i) $\Rightarrow$ (iii) is obvious, we only have to prove (iii) $\Rightarrow$ (i). Let $\pi: S \rightarrow$ $B(H)$ be a finite type representation that is uniformly bounded and unimodular. Denote by $E$ the orthogonal projection on $\operatorname{Ker}\left(R_{\pi}\right)$, where:

$$
R_{\pi}=\int \pi(s)^{*} \pi(s)
$$


Since we have $\pi(s)^{*} R_{\pi} \pi(s)=R_{\pi}$ for any $s \in S$, we get:

$$
\operatorname{Ker}\left(R_{\pi}\right)=\operatorname{Ker}\left(R_{\pi} \pi(s)\right),
$$

a fact which implies that $(1-E) \pi(s) E=0$, i.e. $\pi(s) E=E \pi(s) E$ for any $s \in S$. Let $x \in H$ be fixed, and consider the trace $\tau(X)=\left\langle X^{\#} x \mid x\right\rangle$ on $W^{*}(\pi)$. Since $\pi(s)$ is unimodular, we get by theorem 4 :

$$
\tau(E) \leq \tau\left(E \pi(s)^{*} \pi(s) E\right) \quad \text { for any } \quad s \in S,
$$

and hence:

$$
\left\langle E^{\#} x \mid x\right\rangle \leq\left\langle\left(E \pi(s)^{*} \pi(s) E\right)^{\#} x \mid x\right\rangle .
$$

It follows that:

$$
E^{\#} \leq\left(E \pi(s)^{*} \pi(s) E\right)^{\#}=\left(\pi(s)^{*} \pi(s) E\right)^{\#} \quad \text { for any } \quad s \in S,
$$

and hence $E^{\#} \leq\left(R_{\pi} E\right)^{\#}=0$ by proposition 1(ii). This implies that $E=0$, and $R_{\pi}$ is injective. By proposition 2(iii), we get that $\pi$ is bounded away from 0 , and the conclusion follows from theorem 3 .

Remark 3. Theorem 5 is false if the representation $\pi$ is not of finite type. Indeed, let $V$ be the Volterra integration operator defined on $H=$ $L^{2}([0,1], d x)$ by $V f(x)=\int_{0}^{x} f(y) d y$, and consider the bounded operator $T=(I+V)^{-1}$. Since $\|T\|=1$ and $\operatorname{Sp}(T)=\{1\}$, the representation of the amenable semigroup $S=N$ given by $\pi(n)=T^{n}(n \in N)$ is uniformly bounded and unimodular. However, $\pi$ is not unitarizable because otherwise $T$ would be conjugate to some unitary $U$ with $\operatorname{Sp}(U)=\{1\}$, so that $U=I$ and hence $T=I$, a fact which is absurd. Here, the representation $\pi$ is not of finite type since $V$ is compact with infinite dimensional range.

\section{REFERENCES}

1. Day, M., Amenable semigroups, Illinois J. Math. 1 (1957), 509-544.

2. Dixmier, J., Les moyennes invariantes dans les semi-groupes et leurs applications, Acta. Sci. Math. (Szeged) 12 (1950), 213-227.

3. Dixmier, J., Les algèbres d'opérateurs dans l'espace hilbertien (Algèbres de von Neumann), Gauthier-Villars, Paris (1969).

4. Dodds, P. G., Dodds, T. K. Y., and de Pagter, B., Non-Commutative Banach Function spaces, Math. Z. 201 (1989), 583-597.

5. Dunford, N., and Schwartz, J. T., Linear operators, vol. I: General theory, Intersciences Publishers, Wiley and Sons (1958).

6. Fack, T., Sur la notion de valeur caractéristique, J. Operator Theory 7 (1982), 307-333.

7. Fack, T., Proof of the conjecture of A. Grothendieck on the Fuglede-Kadison determinant, J. Funct. Anal. 50 (1983), 215-228. 
8. Fack, T., and Kosaki, H., Generalized s-numbers of $\tau$-measurable operators, J. Funct. Anal. 50 (1983), 215-228.

9. Glicksberg, I., and de Leeuw, K., Applications of almost periodic compactifications, Acta. Math. 105 (1961), 63-97.

10. Greenleaf, F. P., Invariant means on topological groups, Van Nostrand Mathematical Studies 16 (1969).

11. Hewitt, E., and Ross, K., Abstract Harmonic Analysis, vol. I, Academic Press, New-York, 1963.

12. Van Neerven, J., The asymptotic behaviour of semigroups of linear operators, Oper. Theory, Adv. App. 88 (1996).

13. Russo, B., Unimodular contractions in Hilbert spaces, Pacific J. Math. 26 (1968), 163-169.

INSTITUT GIRARD DESARGUES

UNIVERSITÉ CLAUDE-BERNARD (LYON I)

43, BD DU 11 NOVEMBRE 1918

69622, VILLEURBANNE CEDEX

FRANCE

E-mail: Thierry.Fack@univ-lyon1.fr 